\title{
A Case of Myoepithelioma on the Neck
}

\author{
Hyeree Kim, Hei Sung Kim, Sang Hyun Cho, Jeong Deuk Lee* \\ Department of Dermatology, Incheon St. Mary's Hospital, College of Medicine, The Catholic University of Korea, Seoul, Korea
}

Received: June 15, 2016; Accepted: June 23, 2016; Published: July 01, 2016

*Corresponding author: Jeong Deuk Lee, Professsor, Department of Dermatology, Incheon St. Mary's Hospital, College of Medicine, The Catholic University of Korea, 56 Donsuro, Bupyeong-gu, Incheon, 403-720, Korea. Tel: 82-32-820-5100; Fax: 82-2-506-9514; E-mail: leejd@olmh.cuk.ac.kr

Keywords: Cutaneous Myoepithelioma; Skin Myoepithelioma; Neck Myoepithelioma; Myoepithelioma on the Skin

\section{Case Report}

Cutaneous Myoepithelioma (CM) is a rare, benign, subcutaneous or dermal tumor [1]. The tumor typically presents on the extremities as a small, slow-growing subepidermal mass [1]. Myoepithelioma is comprised solely of myoepithelial cells [2]. Myoepithelial cells are normally seen around the secretory portion of apocrine and eccrine sweat glands in the skin [2]. Only 39 cases have been reported worldwide, with none on the neck [1-5]. Here in we report a rare case of a CM on the neck in a Korean woman.

A 53-year-old woman presented with a tender, solitary, $1.2 \mathrm{~cm}$-sized, dome-shaped, hard subdermal nodule on the neck for 3 years. (Figure 1A, 1B) She denied of previous trauma on the exact site, but had a history of undergoing 4 cycles of transcatheter arterial chemoembolization for hepatocellular carcinoma. We initially suspected benign skin tumors such as epidermal cyst, lipoma, and neuroma etc. When a punch biopsy performed, a yellow mass bulged out. The unencapsulated, but well demarcated mass was completely removed by squeezing. (Figure 1C)

Histopathological evaluation revealed a well-circumscribed dermal nodule consisting of solid nests of uniform epitheloid cell. (Figure 2) On immunohistochemical staining, the cells were positive for EMA and S-100. From the histological findings, a diagnosis of CM was made. The lesion was completely removed during the punch biopsy and the patient is currently well with no signs of recurrence.

Myoepitheliomas are tumors composed of myoepithelial cells without ductal development [4]. Myoepithelial cells are specific contractile cells common in the respiratory tract, breast tissue, salivary glands, and the skin [1-4].

The first CM was identified in 1997 [1]. To our knowledge, there have only been 39 cases of CM and 4 cases of cutaneous myoepithelial carcinoma cases so far [1]. Although the anatomic distribution is broad, these tumors have been most commonly found on the extremities, and have not been described on the neck [1-5].
Vickie et al. published an article about the clinicopathologic characterics of 38 cases of CM between 1997 and 2012 [3]. According to the study, lesions ranged in size from 0.3 to $2.7 \mathrm{~cm}$, the patient's age ranged between 2 months to 74 years with a male preponderance (2.5:1). Most tumors were well circumscribed but lacked a capsule [3]. The anatomic distribution was as follows: upper extremity (11), shoulder (3), lower extremity (15), back (6), face (2), chest (1), and buttock (1). None had been described

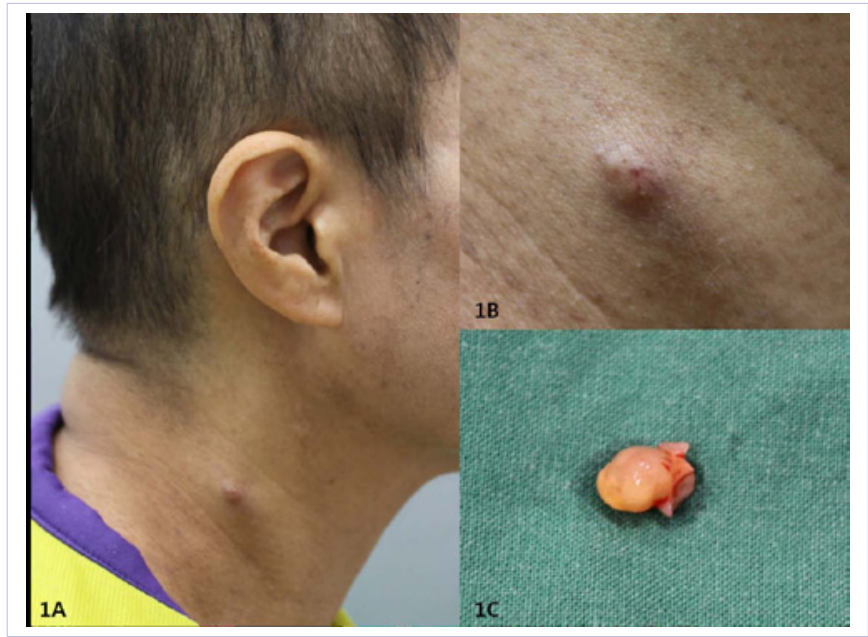

Figure 1: (A) (B): A 1.2cm-sized, dome-shaped, hard subdermal nodule on the neck for 3 years. (C) The yellowish, unencapsulated mass was obtained by squeezing.

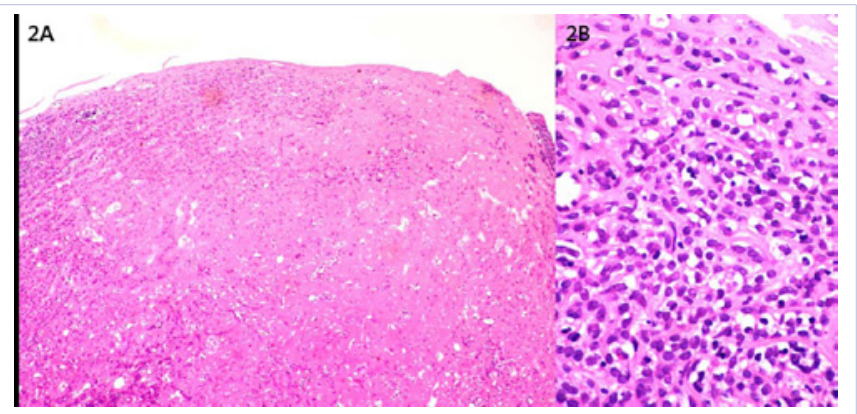

Figure 2: (A) Histopathologic findings revealed a well-circumscribed and unencapsulated mass. (H\&E, x40),

(B) Tumor cells are uniformly sized ovoid to spindled or histiocytoid cells with palelyeosinophilic cytoplasm. (H\&E, x400). 
on the neck [1-5].

Myoepithelial tumors typically coexpressepithelial antigens (keratin, EMA), S-100 protein, Glial fibrillary acidic protein; staining for p63, smooth muscle actin, and calponin is variable [1-5]. As myoepithelial cells originate from the ectoderm, they can display both epithelial and mesenchymal features [2].

CM appears clinically as a cutaneous nodule that gradually increasing in size. Skin biopsies are necessary for confirmation.

The gold standard for treatment of CM is surgical excision [1-5], Mohs micrographic surgery is recommended in case with recurrentor aggressive features [1-5]. Local recurrence and metastases to lymph nodes have been reported in rare cases.

In our case, the tumor presented as a solitary skin nodule which was easily removed by the biopsy and squeezing. There have been no signs of recurrence so far. We believe our case is the first case of a CM to occur on the neck and should be considered as a rare differential diagnosis in benign neck lesions.

\section{References}

1. Brian TD, Matthew JH, Karl RS, Uma K. Cutaneous Myoepithelioma in the Foot: Case Report. Foot Ankle Spec. 2013;6(3):239-241. doi: $10.1177 / 1938640012470713$.

2. Choi YJ, Lee GY, Kim WS. Cutaneous myoepithelioma on the palm. J Dermatol. 2011;38(12):1210-1213. DOI: 10.1111/j.13468138.2011.01255.x

3. Vickie YJ, Cristina RA, Lei Z, Paola DC, Jason LH, Christopher DMF. Cutaneous Syncytial Myoepithelioma: Clinicopathologic Characterization in a Series of 38 Cases. Am J Surg Pathol. 2013;37(5):710-718. doi: 10.1097/PAS.0b013e3182772bba.

4. Stojsic Z, Brasanac D, Boricic I, Bacetic D. Clear cell myoepithelialcarcinoma of the skin: A case report.J Cutan Pathol. 2009;36(6):680-683. doi: 10.1111/j.1600-0560.2008.01095.x

5. Jason LH, Christopher DMF. Cutaneous Myoepithelioma: A Clinicopathologic and Immunohistochemical Study of 14 Cases. Human pathol. 2004;35(1):14-24. 